\title{
SYNTHESIS, ANTIMICROBIAL ACTIVITY AND DOCKING STUDIES OF 1-METHYL-3-(QUINOXALIN-2-YL) PYRROLIDIN-2-ONE
}

\author{
K. Srivani ${ }^{1,2}$, E. Laxminarayana ${ }^{3}$ and M. Thirumala Chary ${ }^{1, \bigotimes}$ \\ ${ }^{1}$ Jawaharlal Nehru Technological University Hyderabad, Kukatpally, Hyderabad, \\ Telangana -500 085 India \\ ${ }^{2} \mathrm{~S}$ R University, Ananthsagar, Hasanparthy, Warangal, Telangana 506371 \\ ${ }^{3}$ Sreenidhi Institute of Science and Technology (Autonomous) Yamnampet, Ghatkesar, \\ Hyderabad- 501301, Telangana, India \\ ${ }^{\circledR}$ Corresponding Author: mtcharya@yahoo.com
}

\begin{abstract}
The preparation of 1-methyl-3-(quinoxalin-2-yl)pyrrolidin-2-onewas described in a simple manner followed by green procedures, title compound was thoroughly characterized by IR, ${ }^{1} \mathrm{HNMR}$ and mass spectra data. The resulted compounds 5, 6 and 7 screened for biological activity and docking studies results are presented.

Keywords: Phosphorous oxychloride, diethylmalonate, Lithium chloride, 2-bromo-1,1-dimethoxyethane

RASĀYAN J. Chem., Vol. 14, No.1, 2021
\end{abstract}

\section{INTRODUCTION}

Quinoxalines exhibits a wide variety of biological activities, because of this the study of quinoxalines became more interesting. The biological activities areantibacterial ${ }^{1-6}$ antifungal $^{7-8}$, anticancer ${ }^{9-11}$, antitubercular $^{12}$, antileishmanial ${ }^{13}$, antimalarial ${ }^{14-15}$ and antidepressant activities ${ }^{16-17}$. The compounds like quinoxalin-2-ones and quinoxaline-2,3-diones have been reported to the activities like antimicrobial ${ }^{18-19}$, potent antithrombotic ${ }^{20}$, analgesic and anti-inflammatory ${ }^{21-22}$ activities.Few synthetic quinoxalines are included in various antibiotics such asactinomycin, echinomycinand levomycin. Some known drugs which contain quinoxaline moiety are Brimonidins, alleviate glaucoma symptoms. ${ }^{23}$<smiles>Brc1c(NC2=NCCN2)ccc2nccnc12</smiles>

Brimonidine

Quinoxalines have a wide range of applications in DNA cleaving agents, dyes, organic semiconductors, dihydroannunulenes, cavitands, and efficient electroluminescent and used as main components for the synthesis of anion receptor. In continuous to our earlier work ${ }^{24}$ and due to this wide range of applications of quinoxalines derivatives in various fields, we synthesized and characterized the novel title derivative of quinoxaline.

\section{RESULTS AND DISCUSSION}

The final compound was prepared as outlined in Scheme-1;Thequinoxalin-2-ol 1 was reacted with $\mathrm{POCl}_{3}$ for 18 hat $80^{\circ} \mathrm{C}$ and obtained good yields of compound 2, which was then treated with diethyl malonate in presence of DMF, $\mathrm{NaH}$ at room temperature to get compound $\mathbf{3}$ with $80 \%$ of yield. Compound 3 was treated with $\mathrm{LiCl}$ in DMSO at room temperature to give compound 4 with $79 \%$ yield. When compound 4 reacted with 2-bromo-1,1-dimethoxyethaneat $0{ }^{\circ} \mathrm{C}$ for $4 \mathrm{~h}$ in presence of $\mathrm{NaH}, \mathrm{DMF}$, 
RASĀYAN J. Chem.

Vol. 14 | No. 1 |82-87| January - March | 2021

it afforded compound $\mathbf{5}$ with $90 \%$ of yield. Compound 5 is reacted with $\mathrm{HCl}$ to formcompound6, which undergoes self-condensation to give title compound 7.

\section{Antibacterial Activity}

The synthesized compounds $(\mathbf{5 ,} \mathbf{6}$ and 7) were screened for their antibacterial activity by agar diffusion method $^{25}$. Among the compounds tested $(5,6,7)$ showed prominent antibacterial activity compared with that from other compounds $5 \mathrm{~g}$ noticed the highest zone of inhibition $16 \mathrm{~mm}$ against gram-negative strain protius vulgaris. Proteus vulgaris is a Gram-negative bacteria with rod-shaped and nitrate-reducing that inhabits the intestinal tracts of humans and animals. These bacteria are present in water, soil, and fecal matter. This bacteria causes wound infections and other species of the same bacterial type are known to cause urinary tract infections. All the gram-negative strains are more susceptible to tested compounds. The zone of inhibition was represented in mm (Table-1).

\section{Docking Studies}

The docking server software was used for docking studies to find out the biointeractions between the ligand and the protein 5-cox oxidoreductase. The compound synthesized was docked to protein 5-cox oxidoreductase. The protein is known for its role in inflammation. The estimated free energy of binding is $-4.89 \mathrm{kcal} / \mathrm{mol}$ which is shown inTable-2. The docking pose of the ligand with the protein 5-cox oxidoreductase showing the binding of the aminoacidsASN68, TYR55, SER38, GLN42, PRO35, LYS166 (Fig.-1). Decomposed interaction energies of different hydrogen, hydrophobic and other bonds in $\mathrm{kcal} / \mathrm{mol}$ are shown in Table-3 which shows the presence of hydrogen, halogen, polar, hydrophobic and other bonds that are involved in this interaction. The interaction of different carbon and nitrogen atoms binding to different amino acids is shown in Table-4. Figure- 2 representing the $2 \mathrm{D}$ plot of the interaction between enzyme and the ligand showing clearly the nearness of the amino acids of the enzyme towards the ligand. Docking observations of the compound in the present scheme indicates that the compound acts as an anti-inflammatory agent.

\section{EXPERIMENTAL}

Chemicals and reagents used in the reaction were obtained from Finar, solvents used are of grade LR. Merck AL silica gel $60 \mathrm{~F}_{254}$ plates were used for thin-layer chromatography (TLC) and these plates were imagined under UV light. ${ }^{1} \mathrm{H}$ NMR spectra and ${ }^{13} \mathrm{C}$ NMR spectra were recorded with a Varian Mercury plus $400 \mathrm{MHz}$ instrument in DMSO- $d_{6}$. By taking TMS as an internal standard Chemical shift are reported in $\delta(\mathrm{ppm})$. Assuming the first-order behavior ${ }^{1} \mathrm{H}-\mathrm{NMR}$ coupling constants and chemical shifts of compounds were determined. Multiplicity is indicated by the following: $d$ (doublet), $t$ (triplet), s (singlet), m (multiplet), br (broad), q (quartet); the list of coupling constants $(J)$ resembles the order of multiplicity assignment. Mass spectra were recorded by using Shimadzu LCMS-QP 1000 mass spectrometer. Open glass capillaries on a Stuart SMP30 apparatus was used to determine the melting points and are uncorrected. The reactions of the scheme were done under an inert atmosphere.
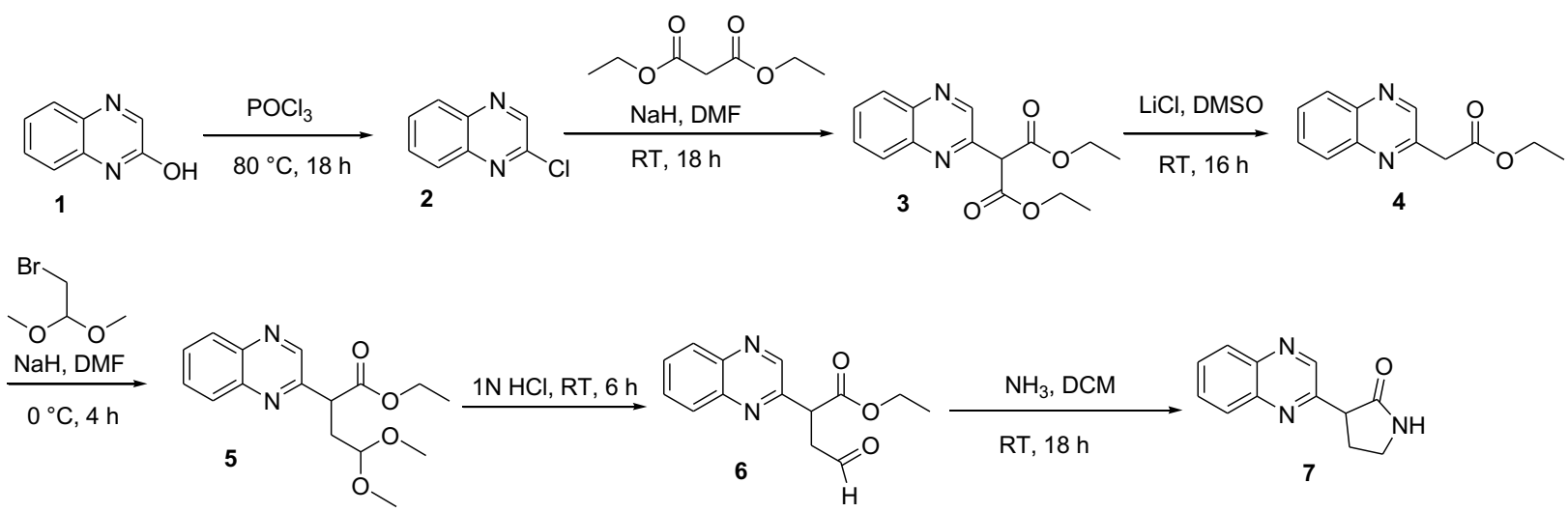

Scheme-1: Synthesis of 1-Methyl-3-(quinoxalin-2-yl)pyrrolidin-2-one 


\section{Diethyl 2-(quinoxalin-2-yl)malonate (3)}

To a suspension of $\mathrm{NaH}(1.5 \mathrm{eq})$ in THF $(10 \mathrm{Vol})$ a solution of diethyl malonate (1 eq.) in DMF (5vol) was added dropwise and stirred the reaction for $1 \mathrm{hr}$ at the same temperature. Then at $0^{\circ} \mathrm{C} 2$ chloroquinoxaline (2) was added, the reaction mixture was allowed to come to room temperature then stirred at $70^{\circ} \mathrm{C}$ for $18 \mathrm{~h}$. TLC technique was used to know the progress of the reaction. The reaction mixture was reduced with sat. $\mathrm{NH}_{4} \mathrm{Cl}$ solution was discharged into ice water and then EtOAc was used for extraction. The extract was washed with water, brine solution, dried over anhydrous $\mathrm{Na}_{2} \mathrm{SO}_{4}$ and evaporated the solvent to afford crude product. Purification of crude product was done by column chromatography; 50\% EtOAc in Petroleum ether was used to extract diethyl 2-(quinoxalin-2-yl) malonate (3)as a white solid.

${ }^{1} \mathrm{H}$ NMR $\left(\right.$ DMSO- $\left._{6}, 400 \mathrm{MHz}\right): \delta=8.92(\mathrm{~s}, 1 \mathrm{H}), 8.10(\mathrm{~d}, 2 \mathrm{H}, J=7.8 \mathrm{~Hz}), 7.75(\mathrm{t}, 2 \mathrm{H}, J=7.8 \mathrm{~Hz}), 4.61(\mathrm{~s}$, $1 \mathrm{H}), 4.18(\mathrm{q}, 4 \mathrm{H}), 1.28(\mathrm{t}, 6 \mathrm{H}) ; \mathrm{MS}: \mathrm{m} / \mathrm{z}, 288.9\left(\mathrm{M}^{+}+\mathrm{H}\right)$.

\section{Ethyl-2-(quinoxalin-2-yl)acetate (4)}

Diethyl-2-(quinoxalin-2-yl) malonate(3) in DMSO (5 vol) mixture was added to $\mathrm{LiCl}(1.2$ eq )at room temperature. Then reaction was heated for $16 \mathrm{~h}$ at $120{ }^{\circ} \mathrm{C}$. After completion of the reaction, the product was identified by TLC. The reaction was quenched with $s a t . \mathrm{NH}_{4} \mathrm{Cl}$ solution. The reaction mixture was poured into ice water, extracted with EtOAc, combined extracts were washed by water, $\mathrm{NaCl}$ solution, dehydrated over anhy. $\mathrm{Na}_{2} \mathrm{SO}_{4}$ and the solvent were evaporated to afford crude

product. Purification of crude product was done by column chromatography; the required product was extracted with 50\% EtOAc in Pet ether to getethyl 2-(quinoxalin-2-yl) acetate(4)as solid.

${ }^{1} \mathrm{H}$ NMR (DMSO-d $\left.6,400 \mathrm{MHz}\right) ; \delta=8.98(\mathrm{~s}, 1 \mathrm{H}), 8.11(\mathrm{~d}, 2 \mathrm{H}, J=7.8 \mathrm{~Hz}), 7.76(\mathrm{t}, 2 \mathrm{H}), 4.20$ (q, 2H), 3.61 $(\mathrm{d}, 2 \mathrm{H}), 1.26(\mathrm{t}, 3 \mathrm{H}) ; \mathrm{MS}: \mathrm{m} / \mathrm{z}, 216.9\left(\mathrm{M}^{+}+\mathrm{H}\right)$.

\section{Ethyl 4-ethoxy-4-methoxy-2-(quinoxalin-2-yl)butanoate (5)}

To a suspension of $\mathrm{NaH}\left(1.5\right.$ eq.) in $\mathrm{DMF}(5 \mathrm{vol})$ at $0^{\circ} \mathrm{C}$ was added solution of ethyl 2-(quinoxalin-2yl)acetate (4) in DMF (2vol) as dropwise. This was stirred for $1 \mathrm{~h}$, and then cooled to $0{ }^{\circ} \mathrm{C}$. To the resulting mixture,2-bromo-1,1-dimethoxyethane (1.2 eq.) was added and stirred at room temperature for 6 h. The reaction mixture was quenched with sat. $\mathrm{NH}_{4} \mathrm{Cl}$ solution, transferred into ice-cold water, extracted with EtOAc. The extract was rinsed with water, $\mathrm{NaCl}$ solution, dried over anhy. $\mathrm{Na}_{2} \mathrm{SO}_{4}$ and evaporated the solvent to getthe crude product. Purification of crude product was done by column chromatography; the required product was eluted with Pet ether with 50\% EtOAc to afford ethyl 4-ethoxy-4-methoxy-2(quinoxalin-2-yl)butanoate (5)as white solid.

${ }^{1} \mathrm{H}$ NMR $\left(\mathrm{DMSO}_{\mathrm{d}}, 400 \mathrm{MHz}\right): \delta=8.97(\mathrm{~s}, 1 \mathrm{H}), 8.12(\mathrm{~d}, 2 \mathrm{H}, J=8.0 \mathrm{~Hz}), 7.78(\mathrm{t}, 2 \mathrm{H}, J=8.0 \mathrm{~Hz}), 4.42(\mathrm{~m}$, 2H), 4.10 (q, 2H), $3.81(\mathrm{t}, 1 \mathrm{H}), 3.60(\mathrm{~s}, 6 \mathrm{H}), 2.31(\mathrm{~m}, 2 \mathrm{H}), 1.12(\mathrm{t}, 3 \mathrm{H}) ; \mathrm{MS}: \mathrm{m} / \mathrm{z}, 305.0\left(\mathrm{M}^{+}+\mathrm{H}\right)$.

\section{Ethyl 3-formyl-2-(quinoxalin-2-yl)propanoate (6)}

To a ethyl 4-ethoxy-4-methoxy-2-(quinoxalin-2-yl)butanoate(5) $1 \mathrm{~N} \mathrm{HCl} \mathrm{(5} \mathrm{vol)} \mathrm{solution} \mathrm{was} \mathrm{added} \mathrm{at} 0^{\circ} \mathrm{C}$ and stirred the reaction for $4 \mathrm{~h}$. The reaction mixture was quenched with sat $\mathrm{NH}_{4} \mathrm{Cl}$ solution, transferred into ice-cold water, extracted with EtOAc. The combined extract was washed with brine solution, water and dried over anhy. $\mathrm{Na}_{2} \mathrm{SO}_{4}$ than the solvent was evaporated to getthe crude product. Column chromatography was used to purify the Crude product; the required product was extracted with $50 \%$ EtOAc in Pet ether to get ethyl 3-formyl-2-(quinoxalin-2-yl) propanoate(6) as off-white solid.

${ }^{1} \mathrm{H}$ NMR (DMSO-d 6 , 400MHz): $\delta=9.79(\mathrm{~s}, 1 \mathrm{H}), 8.71(\mathrm{~s}, 1 \mathrm{H}), 7.84(\mathrm{~d}, 2 \mathrm{H}, J=7.8 \mathrm{~Hz}), 7.70(\mathrm{~m}, 2 \mathrm{H}), 4.20$ $(\mathrm{m}, 2 \mathrm{H}), 4.20(\mathrm{t}, 2 \mathrm{H}), 3.90(\mathrm{~m}, 1 \mathrm{H}), 2.98(\mathrm{~m}, 2 \mathrm{H}), 1.21(\mathrm{t}, 3 \mathrm{H}) ; \mathrm{MS}: \mathrm{m} / \mathrm{z}, 258.9\left(\mathrm{M}^{+}+\mathrm{H}\right)$.

\begin{tabular}{l|l|l|l|l}
\multicolumn{5}{c}{ Table-1 } \\
\hline Compound & 5 & 6 & 7 & Gentamycin \\
\hline \multicolumn{5}{c}{ gram-positive } \\
\hline Bacillussubtitis & 11 & 9 & 8 & 19 \\
\hline Bacilluscereus & 10 & 11 & 7 & 14 \\
\hline S.aureus & 8 & 11 & 10 & 16 \\
\hline
\end{tabular}


RASĀYAN J. Chem.

Vol. 14 | No. 1 |82-87| January - March | 2021

\begin{tabular}{l|l|l|l|l}
\hline \multicolumn{5}{c}{ Gram-negative } \\
\hline E.coli & 9 & 11 & 13 & 13 \\
\hline Protevs vulgaris & 14 & 16 & 11 & 20 \\
\hline k.pneumonia & 6 & 11 & 7 & 15 \\
\hline
\end{tabular}

\section{1-methyl-3-(quinoxalin-2-yl)pyrrolidin-2-one (7)}

To a stirred solution of ethyl 3-formyl-2-(quinoxalin-2-yl)propanoate(6) (1 eq.) in DCM (10 Vol) an amine (B) (1 eq.) was added and stirred the reaction for $18 \mathrm{~h}$ at room temperature. The reaction mixture was poured into ice-cold water and extracted with EtOAc. The extract was washed with water, brine solution, dried over anhy. $\mathrm{Na}_{2} \mathrm{SO}_{4}$ and evaporated the solvent to obtain a crude product. Crude product purification was observed by column chromatography; the required product was eluted with $50 \%$ EtOAc in Pet ether to obtain 1-methyl-3-(quinoxalin-2-yl)pyrrolidin-2-one (7) as a white solid.

${ }^{1} \mathrm{H}$ NMR (DMSO-d $\left.6,400 \mathrm{MHz}\right): \delta=8.72(\mathrm{~s}, 1 \mathrm{H}), 8.18(\mathrm{~d}, 2 \mathrm{H}, J=7.8 \mathrm{~Hz}), 7.70(\mathrm{~m}, 2 \mathrm{H}), 3.92(\mathrm{~m}, 1 \mathrm{H})$, $3.61(\mathrm{~m}, 2 \mathrm{H}), 2.78(\mathrm{~s}, 1 \mathrm{H}), 2.21(\mathrm{~m}, 1 \mathrm{H}), 2.12(\mathrm{~m}, 1 \mathrm{H}) ; \mathrm{MS}: \mathrm{m} / \mathrm{z}, 228.0\left(\mathrm{M}^{+}+\mathrm{H}\right)$.

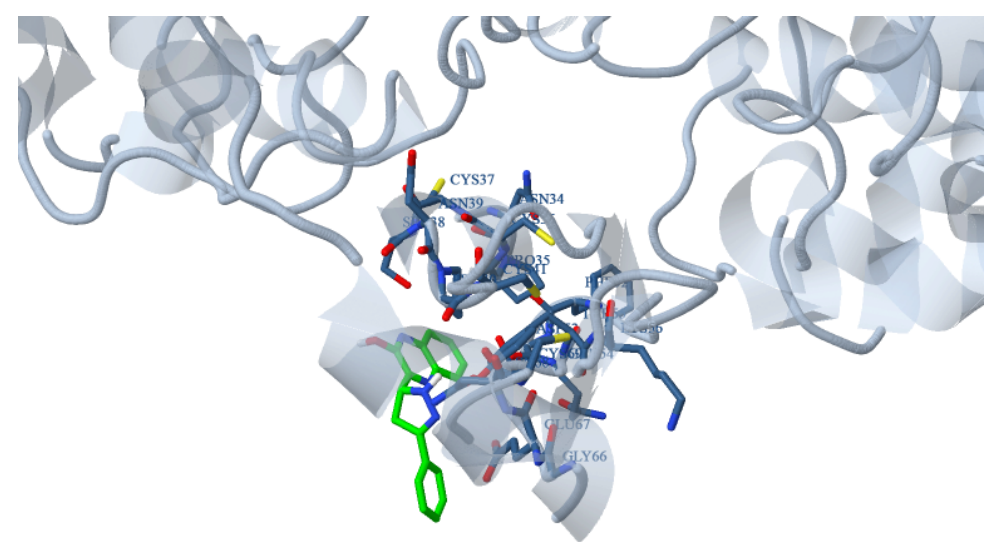

Fig.-1 Interaction of Ligand and Protein
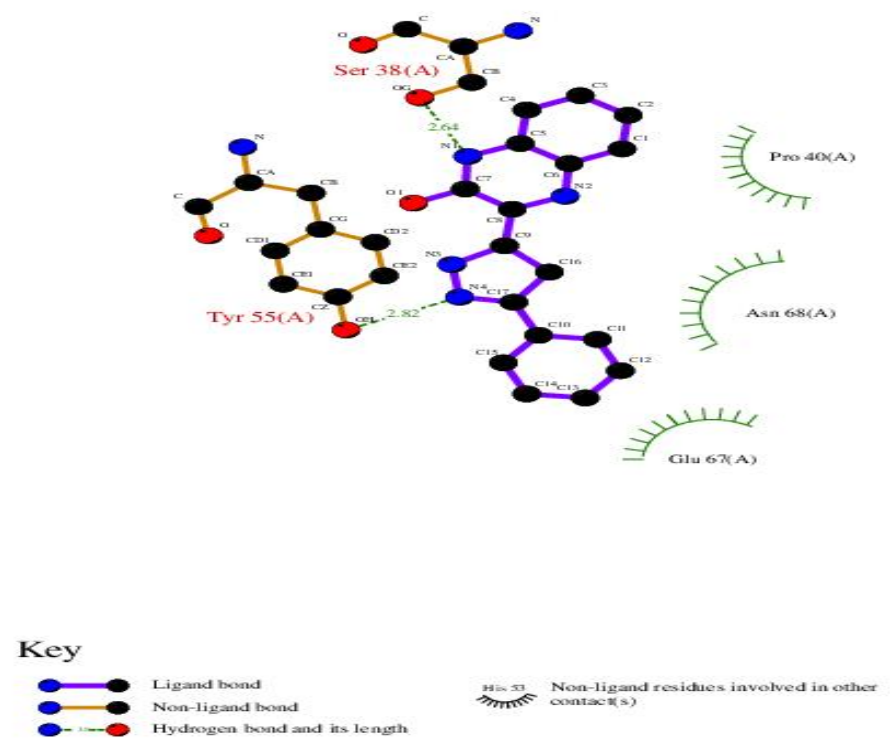

Fig.-2 Docking of Ligand

Table-2: Free Energy of Binding

\begin{tabular}{c|c|c|c|c|c|c}
\hline $\begin{array}{c}\text { Est. Free Energ } \\
\text { y of Binding }\end{array}$ & $\begin{array}{c}\text { Est. Inhibitio } \\
\mathrm{n} \\
\text { Constant, Ki }\end{array}$ & $\begin{array}{c}\mathrm{vdW}+\text { Hbond + desol } \\
\text { v Energy }\end{array}$ & $\begin{array}{c}\text { Electrostati } \\
\mathrm{c} \text { Energy }\end{array}$ & $\begin{array}{c}\text { Total Intermolec } \\
\text {. Energy }\end{array}$ & $\begin{array}{c}\text { Frequenc } \\
\mathrm{y}\end{array}$ & $\begin{array}{c}\text { Interact } \\
. \\
\text { Surface }\end{array}$ \\
\hline$-4.65 \mathrm{kcal} / \mathrm{mol}$ & $389.99 \mathrm{uM}$ & $-5.50 \mathrm{kcal} / \mathrm{mol}$ & $\begin{array}{l}-0.05 \\
\mathrm{kcal} / \mathrm{mol}\end{array}$ & $-5.55 \mathrm{kcal} / \mathrm{mol}$ & $50 \%$ & $\begin{array}{l}414.46 \\
1\end{array}$ \\
\hline
\end{tabular}


RASĀYAN J. Chem.

Vol. 14 | No. 1 |82-87| January - March | 2021

Table-3: Decomposed Interaction Energies in $\mathrm{kcal} / \mathrm{mol}$

\begin{tabular}{c|c|c}
\hline Hydrogen Bonds & Polar & Other \\
\hline SER38 (0) & ASN68 (0) & PRO40 (0) \\
\hline TYR55 (0) & GLU67 (0) & \\
\hline
\end{tabular}

Table-4: Interaction of Ligand and Protein

\begin{tabular}{|c|c|c|c|c|}
\hline Hydrogen Bonds & Polar & pi-pi & Cation-pi & Other \\
\hline $\begin{array}{ll}\text { N1 }() & \text { SER38 } \\
{[2.64]-} & \text { (CB, } \\
& \text { OG) }\end{array}$ & $\begin{array}{l}\mathrm{H} 5() \\
{[3.77]}\end{array}-\begin{array}{l}\text { SER38 } \\
(\mathrm{OG})\end{array}$ & $\begin{array}{ll}\text { C1 }() & \begin{array}{l}\text { TYR55 } \\
{[3.20]}\end{array}-\begin{array}{l}\text { (CD1, } \\
\text { CE1) }\end{array}\end{array}$ & $\begin{array}{ll}\text { H12 }() & \text { TYR55 } \\
{[3.00]} & \text { (CE1, } \\
& \text { CZ) }\end{array}$ & $\begin{array}{ll}\text { C4 () } \\
{[3.61]}\end{array}-\begin{array}{l}\text { SER38 } \\
(\mathrm{OG})\end{array}$ \\
\hline $\begin{array}{ll}\mathrm{O} 10 & \text { SER38 } \\
{[3.41]-} & (\mathrm{CB}, \\
\mathrm{OG})\end{array}$ & $\begin{array}{l}\text { N2 () } \\
{[3.69]}\end{array}-\begin{array}{l}\text { TYR55 } \\
(\mathrm{OH})\end{array}$ & $\begin{array}{l}\text { C2 () } \\
{[3.77]}\end{array}-\begin{array}{l}\text { TYR55 } \\
(\mathrm{CD} 1)\end{array}$ & & $\begin{array}{ll}\text { C5 () } & \text { SER38 } \\
{[3.41]} & (\mathrm{OG})\end{array}$ \\
\hline $\begin{array}{ll}\text { N4 () } & \text { TYR55 } \\
{[2.82]} & (\mathrm{OH})\end{array}$ & 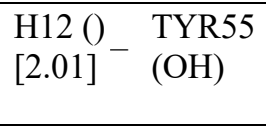 & $\begin{array}{ll}\text { C6 () } & \text { TYR55 } \\
{[3.26]} & (\mathrm{CD} 1, \\
\text { CE1) }\end{array}$ & & $\begin{array}{ll}\text { C7 () } & \text { SER38 } \\
{[3.37]} & (\mathrm{OG})\end{array}$ \\
\hline \multirow[t]{13}{*}{$\begin{array}{ll}\text { N3 () } & \text { TYR55 } \\
{[2.78]} & (\mathrm{OH}) \\
\end{array}$} & $\begin{array}{l}\text { N4 () } \\
{[3.40]}\end{array}-\begin{array}{l}\text { GLU67 } \\
(\mathrm{OE} 2)\end{array}$ & & & 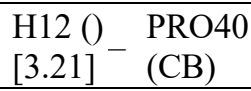 \\
\hline & $\begin{array}{ll}\text { N4 () } & \text { ASN68 } \\
{[2.98]} & (\mathrm{ND} 2, \\
& \text { OD1 }) \\
\end{array}$ & & & $\begin{array}{ll}\text { N2 () } & \text { TYR555 } \\
{[3.29]}\end{array}-\begin{array}{l}\text { (CE1) }\end{array}$ \\
\hline & $\begin{array}{ll}\mathrm{N} 3() & \text { ASN68 } \\
{[3.02]} & (\mathrm{ND} 2, \\
\mathrm{OD} 1)\end{array}$ & & & $\begin{array}{l}\text { N4 () } \\
{[3.56]}\end{array}-\begin{array}{l}\text { GLU67 } \\
(\mathrm{CD})\end{array}$ \\
\hline & $\begin{array}{ll}\mathrm{H} 12() & \begin{array}{l}\text { ASN68 } \\
{[3.06]}\end{array} \\
(\mathrm{ND} 2, & \mathrm{OD} 1)\end{array}$ & & & $\begin{array}{ll}\text { GLU67 } \\
\text { C10 () }- & \begin{array}{l}\text { (CD, } \\
{[3.26]}\end{array} \\
& \text { OE1, } \\
& \text { OE2) } \\
\end{array}$ \\
\hline & & & & $\begin{array}{ll}\text { C11 () } & \begin{array}{l}\text { GLU67 } \\
\text { (CD, } \\
{[3.26]}\end{array} \\
& \text { OE1, } \\
& \text { OE2) }\end{array}$ \\
\hline & & & & $\begin{array}{ll}\text { C12 () } & \text { GLU67 } \\
{[3.37]} & \text { (OE1) }\end{array}$ \\
\hline & & & & $\begin{array}{ll}\text { C13 () } & \text { GLU67 } \\
{[3.45]} & (\mathrm{OE} 1)\end{array}$ \\
\hline & & & & $\begin{array}{ll}\text { C14 () } & \text { GLU67 } \\
{[3.43]} & (\mathrm{OE} 1)\end{array}$ \\
\hline & & & & $\begin{array}{ll}\text { C15 () } & \text { GLU67 } \\
{[3.33]} & (\mathrm{OE} 1)\end{array}$ \\
\hline & & & & $\begin{array}{ll}\text { C17 () } & \text { GLU67 } \\
{[3.76]} & (\mathrm{OE} 2)\end{array}$ \\
\hline & & & & 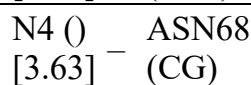 \\
\hline & & & & $\begin{array}{l}\mathrm{N} 3() \\
{[3.60]}\end{array}-\begin{array}{l}\text { ASN68 } \\
(\mathrm{CG})\end{array}$ \\
\hline & & & & $\begin{array}{l}\mathrm{H} 12()-\begin{array}{l}\text { ASN68 } \\
{[3.57]}\end{array}{ }^{(\mathrm{CG})}\end{array}$ \\
\hline
\end{tabular}

\section{REFERENCES}

1. S. Ganapaty, P. Ramalingam, C. B. Rao, Indian Journal of Heterocyclic Chemistry, 16, 283(2007).

2. H. M.Refaat, A. A. Moneer, O. M. Khalil, Archives of Pharmacal Research, 27, 1093(2004), DOI:10.1007/BF02975110

3. M. M. Badran, K. A. M. Abouzid, M. H. M. Hussein, Archives of Pharmacal Research,26, 107(2003). 
RASĀYAN J. Chem.

Vol. 14 | No. 1 |82-87| January - March | 2021

4. M. N. A. Nasr, Journal Pharmaceutical and Medicinal Chemistry, 8, 389(2002), DOI:10.1002/15214184

5. S. A. El-Hawash, N. S. Habib, N. H. Fanaki, Pharmazie, 54, 808(1999).

6. A. A. El-Gendy, S. El-Meligie, A. El-Ansary, A. M. Ahmedy, Archives of Pharmacal Research, 18, 44 (1995).

7. V. K. Tandon, D. B. Yadav, H. K. Maurya, A. K. Chaturvedi, P. K. Shukla, Bioorganic and Medicinal Chemistry, 14, 6120(2006), DOI:10.1016/j.bmc.2006.04.029

8. P. Sanna, A. Carta, M. Loriga, S. Zanetti, L. Sechi, Part 3: II Farmaco, 54, 1169(1999).

9. A. Carta, P. Sanna, Gherardini, D. Usai, ZanettiS, II Farmaco, 56, 933(2001), DOI:10.1016/S0014827X(01)01161-2

10. P. Sanna, A. Carta, M. Loriga, S. Zanetti, L. Sechi, Part 2: II Farmaco, 54, 161(1999).

11. A. Monge, F. J. Martinez-Crespo, A. L. Cerain, J. A. Palop, S. Narro, V. Senador, A. Marin Sainz, M. Gonzalez, E. Hamilton, A. J. Barker, Hypoxia, Journal of Medicinal Chemistry, 38, 4488(1995).

12. A. Jaso, B. Zarranz, I. Aldana, A. Monge, Journal of Medicinal Chemistry, 38, 791(2003), DOI: 10.1371 /journal

13. J. Guillon, I. Forfar, M. M. Matsuda, V. Desplat, M. Saliege, D. Thiolat, Bioorganic and Medicinal Chemistry Letters, 15, 194(2007).

14. J. B. Rangisetty, C. N. V. H. B. Gupta, A. L. Prasad, P. Srinivas, N. Sridhar, P. Parimoo, A. Veeranjaneyulu, Journal of Pharmacy and Pharmacology, 53, 1409(2001), DOI: $10.1211 / 0022357011777765$

15. A. F. Crowther, F. H. S. Curd, D. G. Davey, G. J. Stacey, Journal of Chemical Society, 1260(1949).

16. S.Y. Hassan, S. N. Khattab, A. A. Bekhit, A. Amer, Bioorganic and Medicinal Chemistry Letters, 16(6), 1753(2006), DOI:10.1016/j.bmcl.2005.11.088

17. R. Sarges, H. R. Howard, R. G. Browne, L. A. Lebel, P. A. Seymour, B. K. Koe, Journal of Medicinal Chemistry, 33, 2240(1990), DOI:10.1021/jm00170a031

18. M. M. Ali, M. M. F. Ismail, M. S. A. El-Gaby, M. A. Zahran, Y. A. Ammar, Molecules, 5, 864(2000), DOI:10.3390/50600864

19. C. A. Obafemi, D. A. Akinpelu, Phosphorus, Sulfur, and Silicon and the Related Elements, 180, 1795(2005), DOI: 10.1080/104265090889396

20. U. J. Ries, H. W. Priekpe, N. H. Havel, S. Handschuh, G. Mihm, J.M. Stassen, W. Wienen, NarH, Bioorganic and Medicinal Chemistry Letters, 13, 2297(2003).

21. G. R. Wendt, K. W. Ledig, Chemical Abstract, 70, 106512(1969).

22. D. S. Su, M. G. Bock, US Patent. Appl, 2005, (20050020591).

23. A. Dell, D. H. Williams, H. R. HMorris, Journal of American Chemical Society, 97, 2497(1975), DOI: $10.5923 /$ j.ajoc.20150501.03

24. K. Srivani, T. Thirupathaiah, E. Laxminarayana, M. Thirumala Chary, Rasayan Journal of Chemistry, 11(3), 1004(2018), DOI:10.31788/RJC.2018.1134008

25. N. G. Heatley, Biochemistry Journal, 38, 61(1944), DOI:10.1042/bj0380061

[RJC-5993/2020] 